\title{
Cost-Effectiveness Modeling in Multiple Sclerosis: Playing Around with Non-Healthcare Costs?
}

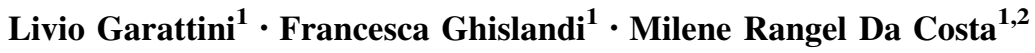

Published online: 21 August 2015

(C) Springer International Publishing Switzerland 2015

\section{Introduction}

Multiple sclerosis (MS) is a chronic disabling neurological disorder that affects more than two million people worldwide [1]. Accounting for more than half of the total population diagnosed with MS in the world, Europe is considered a high-prevalence region [1]. Patients with MS experience a wide range of signs and symptoms [2], which impair their capacity to perform day-to-day activities and thus quality of life [2,3]. The onset of MS at an early age implies a substantial burden in terms of both healthcare and societal costs [2].

The drugs currently used to treat MS aim to alleviate symptoms, delay disease progression and, ultimately, disability. Steroidal anti-inflammatory drugs are traditionally used to inhibit the inflammatory process of MS, then various types of drugs can be used to alleviate the different symptoms (e.g., visual, motor, cognitive). However, the most important therapeutic group is the disease-modifying drugs (DMDs); drugs designed to delay the progression and the development of long-term disability. DMD prescription is still mainly restricted to patients with relapsing-remitting MS (RRMS), although there are some DMDs indicated for specific conditions of secondary progressive MS [4].

Livio Garattini

lgarattini@marionegri.it; livio.garattini@marionegri.it

1 IRCCS Institute for Pharmacological Research "Mario Negri”, Via Camozzi, 3 c/o Villa Camozzi, Ranica, 24020 Bergamo, Italy

2 Faculty of Pharmacy, Federal University of Rio de Janeiro, Rio de Janeiro, Brazil
The two beta interferons (IFN $\beta-1 \mathrm{a}$ and $1 \mathrm{~b}$ ) and glatiramer acetate (GA) have been the first-line DMDs for years. Indicated in patients with at least two relapses in the previous 2 years, they are all injectable (IFN $\beta-1 \mathrm{~b}$ and GA subcutaneous, IFN $\beta$-1a subcutaneous or intramuscular) [4]. More recently, other DMDs have been approved as second-line treatments: (1) natalizumab, a monoclonal antibody injected intravenously; (2) alemtuzumab, another monoclonal antibody for intravenous injection (initially marketed as an anticancer drug) that has recently proved to benefit MS patients, and (3) fingolimod, an oral drug that prevents lymphocyte migration to the central nervous system. Finally, the European Medicines Agency has very recently approved two oral first-line DMDs with different mechanisms of action [5]: teriflunomide and dimethyl fumarate.

The pharmaceutical costs of MS have risen steeply over the previous few years, mainly owing to the introduction of new DMDs. Although medical evidence on the most dated DMDs is quite well established, the economic literature on these drugs is still controversial [6-9], casting doubts on their cost effectiveness. In this commentary, we assess the potential contribution of cost-effectiveness analyses to pricing and reimbursement decisions in Europe. In particular, we analyze the credibility of the major assumptions contained within the analyses.

\section{Economic Evaluations in the European Union}

We searched the PubMed international database to select full economic evaluations on first-generation DMDs (the two IFN $\beta$ s and GA) conducted in European Union (EU) 
countries and published in English from January 2009 until December $2014 .^{1}$

The seven studies finally selected [10-16] came from only four countries (Germany, Italy, Spain, and Sweden) (Table 1); three focused on clinically isolated syndrome $(\mathrm{CIS})^{2}$ (comparing DMD vs. 'do nothing' or early vs. delayed regimens of the same DMD) and the other four on RRMS patients (comparing the two IFN $\beta$ s and GA). Three studies included a cost-utility analysis, two a cost-effectiveness analysis, and the others both. Only one study adopted exclusively the third-party payer's perspective [14]; the others took the societal viewpoint (together with the third-party payer's perspective in two studies). Five studies were based on Markov models with a long-term horizon and two on 'decision trees' $[12,16]$, only one of them with a short-term horizon [16]. All studies used virtual cohorts of patients, only three referring to samples of clinical trials to define the virtual cohorts $[10,11,16]$. Extrapolation of short-term efficacy to long-term time horizons was performed in all studies but one [16]. Six studies sourced most costs from short-term, domestic, prevalence-based cost-of-illness (CoI) studies (all sponsored by DMD manufacturers), which all estimated a wide range of items including indirect and direct non-medical costs. To adapt this information and generate the data lacking to fully populate models, all studies relied on assumptions, three on anonymous experts [10, 11, 15], and two even on non-European [14] or unpublished data [16] to estimate relapse costs. Non-healthcare costs were a substantial proportion of total costs in all the six studies that adopted the societal perspective. All studies were sponsored by the pharmaceutical industry and six were even coauthored by company employees. All studies concluded in favor of the sponsored DMD, their results conflicting depending on the sponsor in the studies on RRMS. Unsurprisingly, time horizons and discount rates were the most influential variables in sensitivity analyses in four of the six studies [10-12, 15] with a long-term horizon.

\footnotetext{
${ }^{1}$ For the search, we used the MeSH terms 'multiple sclerosis' and 'costs and cost analysis'. We retrieved 202 articles and 174 were discarded, being: (1) epidemiological or clinical articles (101); (2) partial economic evaluations and literature reviews (61); and (3) editorials, letters, and comments (12). Because 21 studies did not concern the EU setting, we finally selected seven articles and screened them to assess their main methodologic features, using a common checklist based on the one used to abstract studies in the EURONHEED database.

${ }^{2}$ Frequently, MS begins with isolated neurologic episodes, defined as CIS, which last at least $24 \mathrm{~h}$ and are caused by inflammation and demyelization in focal sites of the central nervous system. CIS can be either monofocal or multifocal and people who experience CIS will not necessarily develop MS.
}

\section{Policy Implications}

Our review of the most recent economic evaluations conducted in the EU setting on first-generation DMDs confirms the widespread concern raised in previous wider reviews [6-9]. First, most studies were based on long-term modeling and they all had to rely on weak sources to populate them [8]. The need for extensive reliance on assumptions is an intrinsic limit of long-term models dealing with clinical efficacy and cost evaluation where only short-term experimental data are available. This is likely to lead to great within- and between-study variability generated by authors' choices of sources of information and assumptions. Then too, the societal viewpoint adopted by most studies seems to play a large role in determining the cost-effectiveness ratio. In fact, almost all the studies sourced costs from CoIs that estimated a substantial proportion of non-healthcare costs in MS, whose evaluation and monetization is open to the authors' discretion [7]. All these CoIs used the 'human capital approach' to assess indirect cost, a controversial method that is likely to lead to overestimates and implies a state of full employment in the long run [17], which is hardly the case in the EU countries in this period of unprecedented economic crisis. Last but not least, all the studies gave the clear impression of being part of the manufacturers' marketing strategies, trying to highlight high social costs for MS to demonstrate the value of their product [7-9].

To sum up, we feel the studies reviewed can be considered no more than mere forecasting exercises, in which short-term information is projected into the future on the basis of heterogeneous estimates and non-healthcare costs are included to enhance the chances of DMDs being cost effective. Because we are reasonably sure that companies will manage to show an acceptable incremental cost-effectiveness ratio for each of the new expensive DMDs despite their high prices, we wonder whether future similar studies will really add any value for public decision making. We would really prefer economic evaluations in this field to be more pragmatic, i.e., conducted from the thirdparty payer's perspective and excluding non-healthcare costs to make their results more plausible, as repeatedly suggested by the National Institute for Health and Care Excellence starting from its very first guidance on DMDs [18-21]. We are also convinced that simpler short-term analyses could be more useful for public decision making to assess the different healthcare costs for new and old DMDs, which all have different mechanisms of action and can be grouped only by form (oral or injectable). Surely, there could be scope for referring more often to 'budget impact analyses' [22] in this field too, to assess whether the new DMDs induce some trade-offs on other healthcare 


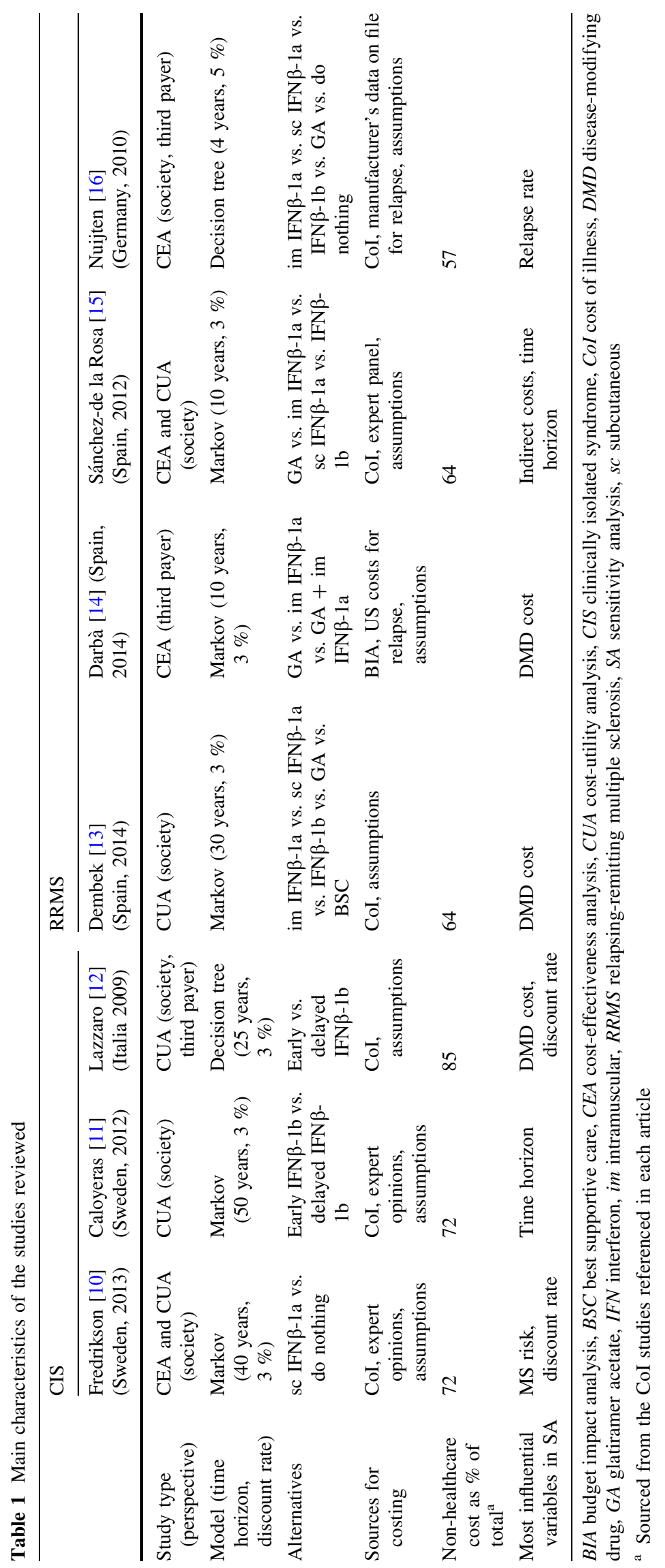


resources (e.g., specialist consultations and nursing interventions) to somehow compensate the extra costs due to their higher prices.

\section{References}

1. Kingwell E, Marriott JJ, Jetté N, Pringsheim T, Makhani N, Morrow S, et al. Incidence and prevalence of multiple sclerosis in Europe: a systematic review. BMC Neurol. 2013;13:128.

2. Zwibel HL. Contribution to impaired mobility and general symptoms to the burden of multiple sclerosis. Adv Ther. 2009;26:1043-57.

3. Compston A, Coles A. Multiple sclerosis. Lancet. 2002;359(9313):1221-31.

4. National Health Service (NHS). Multiple sclerosis, overview. Available from: http://www.nhs.uk/Conditions/Multiple-Sclerosis/ Pages/Introduction.aspx. Accessed 5 Aug 2015.

5. Hutchinson M. In the coming year we should abandon interferons and glatiramer acetate as first line therapy for MS: commentary. Mult Scler. 2012;19(1):29-30.

6. Hawton A, Shearer J, Goodwin E, Green C. Squinting through layers of fog: assessing the cost effectiveness of treatments for multiple sclerosis. Appl Health Econ Health Policy. 2013;11(4):331-41.

7. Thompson JP, Abdolahi A, Noyes K. Modelling the cost effectiveness of disease-modifying treatments for multiple sclerosis: issues to consider. Pharmacoeconomics. 2013;31(6):455-69.

8. Manouchehrinia A, Constantinescu CS. Cost-effectiveness of disease-modifying therapies in multiple sclerosis. Curr Neurol Neurosci Rep. 2012;12(5):592-600.

9. Guo S, Pelligra C, Saint-Laurent Thibault C, Hernandez L, Kansal A. Cost-effectiveness analyses in multiple sclerosis: a review of modelling approaches. PharmacoEconomics. 2014;32(6):559-72.

10. Fredrikson S, McLeod E, Henry N, Pitcher A, Lowin J, Cuche M, et al. A cost-effectiveness analysis of subcutaneous interferon beta-1a 44 mcg 3-times a week vs no treatment for patients with clinically isolated syndrome in Sweden. $\mathrm{J}$ Med Econ. 2013;16(6):756-62.

11. Caloyeras JP, Zhang B, Wang C, Eriksson M, Fredrikson S, Beckmann $\mathrm{K}$, et al. Cost-effectiveness analysis of interferon beta-
$1 \mathrm{~b}$ for the treatment of patients with a first clinical event suggestive of multiple sclerosis. Clin Ther. 2012;34(5):1132-44.

12. Lazzaro C, Bianchi C, Peracino L, Zacchetti P, Uccelli A. Economic evaluation of treating clinically isolated syndrome and subsequent multiple sclerosis with interferonbeta-1b. Neurol Sci. 2009;30(1):21-31.

13. Dembek C, White LA, Quach J, Szkurhan A, Rashid N, Blasco MR. Cost-effectiveness of injectable disease-modifying therapies for the treatment of relapsing forms of multiple sclerosis in Spain. Eur J Health Econ. 2014;15(4):353-62.

14. Darbà J, Kaskens L, Sánchez-de la Rosa R. Cost-effectiveness of glatiramer acetate and interferon beta-1a for relapsing-remitting multiple sclerosis, based on the CombiRx study. J Med Econ. 2014;17(3):215-22.

15. Sánchez-de la Rosa R, Sabater E, Casado MA, Arroyo R. Costeffectiveness analysis of disease modifying drugs (interferons and glatiramer acetate) as first line treatments in remitting-relapsing multiple sclerosis patients. J Med Econ. 2012;15(3):424-33.

16. Nuijten M, Mittendorf T. A health-economic evaluation of disease-modifying drugs for the treatment of relapsing-remitting multiple sclerosis from the German societal perspective. Clin Ther. 2010;32(4):717-28.

17. Drummond M. Cost-of-illness studies: a major headache? Pharmacoeconomics. 1992;2(1):1-4.

18. NICE technology appraisal guidance 32. Beta interferon and glatiramer acetate for the treatment of multiples clerosis. National Institute for Health and Care Excellence (NICE). 2002. Available from: http://www.nice.org.uk/guidance/ta32. Accessed 6 Aug 2015

19. NICE technology appraisal guidance 254. Fingolimod for the treatment of highly active relapsing-remitting multiple sclerosis. National Institute for Health and Care Excellence (NICE). 2012. Available from: https://www.nice.org.uk/guidance/ta254. Accessed 6 Aug 2015.

20. NICE technology appraisal guidance 320. Dimethyl fumarate for treating relapsing-remitting multiple sclerosis. National Institute for Health and Care Excellence (NICE). 2014. Available from: https://www.nice.org.uk/guidance/ta320. Accessed 6 Aug 2015.

21. NICE technology appraisal guidance 303. Teriflunomide for treating relapsing-remitting multiple sclerosis. National Institute for Health and Care Excellence (NICE). 2014. Available from: http://www.nice.org.uk/guidance/ta303. Accessed 6 Aug 2015.

22. Garattini L, van de Vooren K. Budget impact analysis in economic evaluation: a proposal for a clearer definition. Eur J Health Econ. 2011;12:499-502. 